\title{
Enhanced Healing and Antimicrobial Efficacy of Chitosan-g-Polyacrylamide in a Rat Model of Gingival Ulcers
}

\author{
Yanfen Zheng ${ }^{1 * \dagger}$, Lingjie $\mathrm{Ke}^{2 \dagger}$, Yin $\mathrm{Lu}^{1 *}$, Qiliang Zuo ${ }^{1}$, Guanhong Deng ${ }^{1}$, Hairui Wang ${ }^{1}$ and \\ Xiamei Zeng ${ }^{1}$
}

1 Department of Oral Mucosal Diseases and Department of Prosthodontics, Stomatological Hospital of Xiamen Medical College, Xiamen, China, ${ }^{2}$ Fujian Provincial Key Laboratory of Innovative Drug Target Research and State Key Laboratory of Cellular Stress Biology, School of Pharmaceutical Sciences, Xiamen University, Xiamen, China

\section{OPEN ACCESS}

Edited by:

Zibiao Li,

Technology and Research (A*STAR), Singapore

Reviewed by:

Yun-Long Wu,

Xiamen University, China

Dai-xu Wei,

Northwest University, China

${ }^{*}$ Correspondence: Yanfen Zheng

zhengyanfen8210@163.com

Lu Yin

yin/u7989@hotmail.com

tThese authors have contributed equally to this work

Specialty section:

This article was submitted to Polymer Chemistry,

a section of the journal

Frontiers in Chemistry

Received: 13 December 2019 Accepted: 20 March 2020 Published: 24 April 2020

Citation:

Zheng Y, Ke L, Lu Y, Zuo Q, Deng G, Wang $H$ and Zeng $X$ (2020) Enhanced Healing and Antimicrobial Efficacy of Chitosan-g-Polyacrylamide in a Rat Model of Gingival Ulcers.

Front. Chem. 8:273.

doi: 10.3389/fchem.2020.00273
Patients in dental hospitals often experience oral ulcerative lesions, which lead to pain and affect the patient's quality of life. At present, the goal of treating oral ulcerative lesions with drugs is to reduce inflammation and promote ulcer healing. However, very few antibacterial and hemostatic drugs are designed to be suitable for the microenvironment of gingival ulcers. Based on this, we have designed a natural therapeutic agent for oral ulcerative lesions that meets the various requirements of oral ulcerative lesion medication. The chitosan-g-polyacrylamide (CP) copolymer is composed of chitosan as the main chain and polyacrylamide polymers as the side chains. Antibacterial experiments show that this polymer can effectively inhibit the proliferation of Gram-negative (Escherichia coli) and Gram-positive bacteria (Staphylococcus aureus). In vitro cell experiments also show that the CP copolymer is non-toxic, which is conducive to ulcer wound healing. Coagulation experiments prove that the CP copolymer can accelerate blood coagulation to stop bleeding. In experiments using a Wistar rat gingival ulcer model, the CP copolymer significantly promoted ulcer healing and shortened the healing time. These results indicate that the $\mathrm{CP}$ copolymer may serve as a potential therapeutic agent for oral ulcerative lesions.

Keywords: oral, ulcerative lesion, anti-bacterial, chitosan, polyacrylamide

\section{INTRODUCTION}

Oral ulcerative lesions result in a loss of epithelial tissue in the oral mucosa membrane and are often seen in patients in dental hospitals (Trautwein-Weidner et al., 2015). Recurrent aphthous ulcers are considered to be the most common oral mucosa disease, with an incidence rate ranging from 5 to $60 \%$ in the general population (Warinner et al., 2014). Radiation stomatitis is a radiationinduced disease that generally occurs in patients who have received radiotherapy for head or neck cancer. Severe radiation stomatitis can be seen in 29 to $66 \%$ of all head and neck cancer patients after undergoing radiotherapy (Sumita et al., 2014). The extremely painful symptoms associated with these ulcerative diseases may lead to difficulty in chewing, swallowing, and speaking. Such functional limitations may negatively influence the patient's quality of life (Lee et al., 2016). They are often accompanied by halitosis, nausea, chronic pharyngitis, lymph node swelling, and other complications (McGuire et al., 2013). 
Treatments for chronic oral wounds require effective adherence to the wound surface and the ability to prevent infection and promote wound healing. Thus, the therapeutic materials used for this purpose must display appropriate mechanical and swelling properties. At present, oral ulcerative lesion treatments are primarily developed to relieve pain, reduce inflammation, promote ulcer healing, and prolong the attack interval (Vieira et al., 2012) In addition to basic oral care, medications for oral ulcerative lesions include growth factors, anti-inflammatories, cytokines, glucocorticoid, and adrenal hormones (Raber-Durlacher et al., 2013). However, the efficacy of therapeutic drugs may be affected by muscle movement and the continuous secretion of oral saliva, resulting in a low effective concentration of the drugs. With the continuous progress that is being made in mucous membrane adhesives, drug delivery carriers, permeability enhancers, and other technologies, the ability to implement safe and effective drug delivery to treat local or systemic diseases has increased. The progress made in oral biomaterial research has the potential to improve the therapeutic effect of oral ulcerative lesion drugs and decrease drug waste (Martín et al., 2015). Silver nanoparticles, which are natural materials, have been used in wound dressings to defend against local wound infections. However, silver is a strong preservative, and its inevitable toxicity will cause varying degrees of damage to the human body (Wilkinson et al., 2011; Karim et al., 2016). Thus, the development of a biocompatible drug delivery carrier is clearly needed for ulcerative lesions.

Chitosan (CS) is a N-deacetylated derivative of chitin that can be effectively degraded into non-toxic, absorbable monosaccharides that are not teratogenic (Bonferoni et al., 2009; Elsabee et al., 2009). It is the second-most naturally abundant biodegradable polymer material and is used in the biomedicine industry due to its antibacterial, antiinflammatory, anti-thrombotic, hemostatic, wound healing, and immunomodulating effects (Wang and Wang, 2011; Lewandowska, 2015). In recent years, chitosan-grafted polymers have been used in cell stents and wound healing polymer dressings in the biomedicine field (Nettles and Elder, 2002; Berger et al., 2004; Kalambettu and Rajangam, 2012). The surface affinity structure of the double electron layer formed by chitosan contact with mucosal epithelial cells, along with its swelling properties, aids in its adhesion in the oral environment. Chitosan water absorption relaxes the polymer chain from a crimped to a flat state, which increases structural exposure to further increase adhesion.

Although it has many advantages, its poor water solubility significantly limits its use in biological applications. However, the water solubility and biological activity of chitosan can be improved through chemical modification. Mahattanadul et al. found that a $0.1 \%$ chitosan turmeric mouthwash can be used as a potential local therapeutic agent for oral inflammationtype ulceration and anti-candidiasis (Mahattanadul et al., 2018). Zhang et al. constructed a Matrine/CS bio-film with biodegradable chitosan as the carrier material, which displayed good antibacterial performance, biocompatibility, and anti-inflammatory properties during healing of the aphtha (Zhang C. et al., 2019). The clinical trial results of
Shao et al. showed that chitosan can effectively promote drug penetration, robustly adheres to oral mucosa epithelial cells, has a strong curative effect, and can be applied as an excellent medicine for treating oral ulcerative lesions (Shao and Zhou, 2019).

Polyacrylamide (PAM) is a hydrophilic, synthetic anion/nonionic polymer with an active amino group along the main chain. Recent studies have shown that the scaffold structure formed by PAM polymers can effectively promote the proliferation of fibroblasts with a low level of cytotoxicity (Risbud and Bhonde, 2000; Zhang et al., 2005; Lewandowska, 2007). Grafting PAM onto the chitosan backbone increases polymer stability, promotes fibroblast proliferation, and accelerates ulcer healing, making chitosan a viable material for therapeutic applications (Feller et al., 2010). Some studies have shown that the presence of a carbodiimide moiety can mediate the formation of an amine bond between PAM and another polymer, such as chitosan, to form a copolymer conjugate system. In addition, free-radical polymerization can be used to graft PAM onto chitosan using glutaraldehyde or tetraethylene acrylate as a cross-linking agent (Kandow et al., 2007; Sheng et al., 2014).

In this study, we grafted polyacrylamide to chitosan [chitosang-polyacrylamide (CP) copolymer] to increase its solubility for the treatment of oral ulcerative lesions. The CP copolymer was fabricated by conjugating chitosan with several polyacrylamide side chains. The ${ }^{1} \mathrm{H}$ NMR and FTIR spectra indicated that polyacrylamide was effectively grafted onto the main chain of chitosan by in situ free-radical polymerization. We further determined its hemostatic effect, antibacterial activity, and its effect on gingival fibroblast proliferation. An oral gingival ulcer mucous membrane model established using Wistar rats was used to investigate the therapeutic effect of the CP copolymer system. Results showed that the CP copolymer system effectively promoted coagulation, bacterial resistance, and promoted gingival fibroblast proliferation. These findings have not previously been reported and suggest that chitosan is a promising therapeutic agent for oral ulcerative lesions.

\section{EXPERIMENTAL METHODS}

\section{Chemical Materials}

Acrylamide (AM, >98\%), ammonium persulfate (APS, >98\%), acetic acid (>99.5\%), and ethanol were purchased from Sigma-Aldrich Co., Ltd. (Canada). $N, N, N^{\prime}, N^{\prime}$-tetramethyl- 1,2ethanediamine (TEMED, >98\%), Chitosan (CS, deacetylation value, $75-85 \%)$, and sodium hydrate $(\mathrm{NaOH})$ were obtained from TCI (Tokyo Chemical Industry Co., Ltd, Japan). Tryptone and yeast extract were obtained from OXOID Co., Ltd (USA). Agar Powder was purchased from Solarbio Co., Ltd (China). Sodium chloride ( $\mathrm{NaCl}, 99.9 \%)$ was obtained from JHD Co., Ltd (China). DMEM/F-12 culture medium, trypsin-EDTA (0.25\%), penicillinstreptomycin, cell counting kit-8 (CCK-8), and the hematoxylin and eosin $(\mathrm{H} \& \mathrm{E})$ staining kit were all obtained from Yeasen Biotech Co., Ltd (China). Fetal bovine serum (FBS, Gibco) was purchased from Thermo Fisher Co., Ltd (USA). Chloral hydrate, $\mathrm{D}_{2} \mathrm{O}$, and $\mathrm{DCl}$ was obtained from Macklin Biochemical Co. Ltd (China). 


\section{Synthesis Route of CP Copolymer}

Based on our recently reported work with slight modifications, the chitosan-based polymer was synthesized using an in-situ polymerization method in dilute acetic acid (Zhang C. et al., 2019). Chitosan (0.4 g) was dispersed in a dilute acetic acid aqueous solution $(1 \%, 20 \mathrm{~mL})$ and stirred violently at room temperature for $4 \mathrm{~h}$. The insoluble substances were removed by filtration. Then the AM monomer (AM:CS = 7:2 mass ratio) was added to the reaction solution and continuously stirred under $\mathrm{N}_{2}$ for $\sim 20 \mathrm{~min}$ at room temperature. APS and TEMED catalysts (1:1 molar ratio at $3 \mathrm{~mol} \%$ of the monomer concentration) were then added to the solution and stirred for $10 \mathrm{~min}$. Subsequently, the mixture was heated to $30^{\circ} \mathrm{C}$ for $24 \mathrm{~h}$. The solution $\mathrm{pH}$ was adjusted to 9.0 with a $\mathrm{NaOH}$ solution $(1 \mathrm{M})$ to obtain the precipitant. The addition of a few milliliters of ethanol further facilitated precipitation. This purification process was repeated twice. After that, the system was dialyzed for 3 days at room temperature to eliminate unreacted substances (spectrum dialysis membrane, MWCO $10 \mathrm{kDa}$ ). This solution was then frozen and dried to obtain a light-yellow solid. The yield of the CP copolymer was $\sim 85 \%$.

\section{Characterizations of the CP Copolymer by FTIR and 1H NMR}

FTIR spectra were recorded using a $\mathrm{KBr}$ pellet $(4,000-400$ $\mathrm{cm}^{-1}$ ) on a Perkin-Elmer FTIR2000 spectrometer (PerkinElmer Co., Ltd, UK). An average of 64 scans were taken at room temperature with a resolution of up to $2 \mathrm{~cm}^{-1}$. The coupling sample was dried in a vacuum at $50^{\circ} \mathrm{C}$ for 1 day before testing. ${ }^{1} \mathrm{H}$ NMR spectra of conjugates at room temperature were measured with a JEOL ECA $500 \mathrm{MHz}$ NMR spectrometer (JEOL Co., Ltd, Japan). $\mathrm{D}_{2} \mathrm{O}$, containing $0.1 \mathrm{M} \mathrm{DCl}$, was used as the solvent. The chemical shifts were reported in $1 / 1,000,000$ (ppm). The solvent peak of $\mathrm{D}_{2} \mathrm{O}$ is $\delta \mathrm{H} 4.70$.

\section{Antibacterial Behavior of the CP Co polymer}

The antibacterial effects of the CP copolymer were investigated using an inhibition zone method (Lu et al., 2017). Staphylococcus aureus $(1,000,000 \mathrm{CFU} / \mathrm{mL})$ and Escherichia coli $(1,000,000$ $\mathrm{CFU} / \mathrm{mL}$ ) cultures were evenly coated on a solid LB medium plates. Circular copolymer samples (with a diameter of $6 \mathrm{~mm}$ ) were then deposited on the surface of the solid medium; the plates were then incubated at $37^{\circ} \mathrm{C}$ for $24 \mathrm{~h}$. The antibacterial effect of the CP copolymer at different concentrations was evaluated by observing the size of the inhibition zones around the hydrogel samples.

\section{Cell Culture}

Human gingival fibroblasts (HGF-1, Shanghai Sixin Biotech.Co., Ltd., China) were cultured in a cell incubator with DMEM/F12 media, FBS (10\%), and penicillin-streptomycin $(100 \mu \mathrm{g} / \mathrm{mL})$ with $5 \% \mathrm{CO}_{2}$ at $37^{\circ} \mathrm{C}$.

\section{Cell Viability Assay}

The viability of HGF-1 cells incubated with the CP copolymer was investigated using a CCK-8 assay. Cells were cultured in sterile 96 -well plates at $4.8 \times 10^{5}$ cells $/ \mathrm{mL}\left(4.8 \times 10^{4}\right.$ cells per hole) with different concentrations of the $\mathrm{CP}$ copolymer for $24 \mathrm{~h}$. Next, $10 \mu \mathrm{L}$ volume of CCK-8 reagent was added to each well, and sterile 96-well plates were incubated for another $3 \mathrm{~h}$. The absorbance of the samples at a wavelength of $450 \mathrm{~nm}$ was measured using an enzyme labeling instrument (Perkin Elmer Co., Ltd, USA).

\section{Cell Growth Evaluation}

The cell growth evaluation of HGF-1 cells was determined using the CCK-8. When the number of HGF-1 cells reached $2.88 \times$ $10^{5}$ cells $/ \mathrm{mL}$, the cells were cultured in sterile 96-well plates with different concentrations of the CP copolymer for $24 \mathrm{~h}$. Fresh media was replaced every day. Next, $960 \mu \mathrm{L}$ of CCK- 8 was added to the 96 -well plates at 12,24 , and $48 \mathrm{~h}$ of culture and sterile 96 well plates were incubated for $4 \mathrm{~h}$. The absorbance wavelength (450 nm) was measured with an enzyme labeling instrument, and cell growth evaluation were calculated for each time point from the average OD values.

\section{Establishment of Animal Models}

Filter paper $(3 \mathrm{~mm} \times 3 \mathrm{~mm})$ samples with $20 \mu \mathrm{L}$ of a $70 \%$ acetic acid solution were applied (for $1 \mathrm{~min}$ ) to the gingival mucosa location of healthy mature Wistar rats (Shanghai SLAC Laboratory Animal Co.,Ltd, China) that were anesthetized with $10 \%$ chloral hydrate. Application of $70 \%$ acetic acid was repeated for two consecutive days to establish the animal model. Animal treatment procedures and feeding were conducted as per the Xiamen University Animal Care Guidelines.

\section{Analysis of the Hemostatic Effect of the CP Copolymer}

The blood of Wistar rats was collected by retro-orbital extraction into EP tubes that contained heparin. The blood was mixed with the CP copolymer samples (2:1 volume ratio) and incubated in a water bath at $37^{\circ} \mathrm{C}$. Untreated rat blood was used as the control group.

\section{In vivo Effects of the CP Copolymer on Gingival Mucosal Ulcers}

After animal models were established, $20 \mu \mathrm{L}$ of a $5 \mathrm{wt} \% \mathrm{CP}$ copolymer solution was applied to the festering site in the oral cavity of anesthetized rats [chloral hydrate (10\%)] every day. Each experimental group contained three rats, and pictures were taken every 5 days with a camera to measure and record the length and width of the oral cavity using Vernier calipers.

\section{Tissue Section Analyses}

After 10 days of administration, the rats were sacrificed, and tissues were taken isolated and immersed in 15 or $30 \%$ sucrose solutions for $24 \mathrm{~h}$. The tissues were frozen and sliced into sections of $7 \mu \mathrm{m}$ in thickness, then stained with an H\&E stain for microscopic observation. All animal treatment procedures and 
feeding were conducted following the Xiamen University Animal Care Guidelines.

\section{Statistical Analyses}

All experimental data are represented as the mean \pm standard deviation. All experimental data and figures were analyzed using GraphPad Prism 5.0 and OriginPro 8. Statistical significance was indicated when the $p$-value was $<0.05$.

\section{RESULTS AND DISCUSSION}

\section{Synthesis and Characterization of the CP Copolymer}

Our recent work presented a simple, one-pot procedure to prepare chitosan conjugate compounds (Zhang C. et al., 2019; Zhang Z. et al., 2019). The CP copolymer was prepared by in-situ free-radical-induced polymerization using TEMED as an accelerator and APS as an initiator in an acetic acid solution (Figure 1). The sulfate radical produced by thermal decomposition of the APS initiator caused AM polymerization to create a PAM long chain structure, which further polymerized with chitosan in situ (Huang et al., 2013; Echeverria et al., 2015; Wang and $\mathrm{Yu}, 2015)$. CP copolymers were synthesized with a mass ratio of 3.41:1 (PAM:CS). The characteristic chemical shift peaks of PAM and chitosan could both be observed in the ${ }^{1} \mathrm{H}$ NMR spectra of the CP copolymer (Figure S1). The specific characteristic peaks were as follows: $2.38-1.10 \mathrm{~m}$ (protons of the main chain, br); $0.97 \mathrm{~m}\left[-\mathrm{CH}\left(\mathrm{CH}_{3}\right)_{2}\right.$, br] belong to the PAM polymer; 4.12-2.77 m (protons, br), 2.28-1.98 m [- $\mathrm{CH}_{2}-$ $\mathrm{CH}\left(\mathrm{CONH}_{2}\right)-$, br], 1.93-1.88 m (-NHCO- $\mathrm{CH}_{3}$, br), and 1.79$1.21 \mathrm{~m}\left[-\mathrm{CH}_{2}-\mathrm{CH}\left(\mathrm{CONH}_{2}\right)-\right.$, br] belonging to chitosan. The mass ratio (calculated by signal integration from the ${ }^{1} \mathrm{H}$ NMR spectra) of PAM and chitosan in the conjugates was close to that of the initial ratio of monomer to chitosan. The monomer conversion rate of PAM was typically higher than $99 \%$, and the conjugation yield was higher than $85 \%$. The above data indicated that the polymer was grafted onto the chitosan backbone.
The FTIR spectra of conjugates and chitosan were collected to determine the extent of PAM grafting onto the chitosan backbone (Figure S2). The characteristic peaks of chitosan were seen at $1,081 \mathrm{~cm}^{-1}[\mathrm{~s}, v(\mathrm{C}-\mathrm{O}-\mathrm{C})$ and $v(\mathrm{C}-\mathrm{O})], 1,650 \mathrm{~cm}^{-1}[\mathrm{~s}$, $\nu(\mathrm{C}=\mathrm{O})], 2,879 \mathrm{~cm}^{-1}\left[\mathrm{~m}, \nu_{\mathrm{s}}(\mathrm{C}-\mathrm{H})\right.$ and $\left.\nu_{\mathrm{as}}(\mathrm{C}-\mathrm{H})\right]$, and 3,440 $\mathrm{cm}^{-1}$ [strong and wide, br, $v(\mathrm{O}-\mathrm{H})$ and $\nu(\mathrm{N}-\mathrm{H})$ ]. The FTIR spectrum of the CP copolymer also showed additional peaks at $1,452 \mathrm{~cm}^{-1}[\mathrm{~m}, v(\mathrm{C}-\mathrm{N})]$ due to the amide group of PAM, $1,658 \mathrm{~cm}^{-1}\left[\mathrm{~s}, v(\mathrm{C}=\mathrm{O}), 2,930 \mathrm{~cm}^{-1}\left(\mathrm{~m}, \nu_{\mathrm{as}}(\mathrm{C}-\mathrm{H})\right]\right.$, and 3,000$3,750 \mathrm{~cm}^{-1}$ [br, $v(\mathrm{O}-\mathrm{H})$ and $\left.v(\mathrm{~N}-\mathrm{H})\right]$ due to the amide groups of chitosan and PAM. These FTIR results further indicated that PAM grafting onto the chitosan backbone was achieved by in situ free-radical polymerization.

\section{Antibacterial Effects of the CP Copolymer}

Oral bacterial infections often increase the burden of ulcer wound healing, necessitating effective antibacterial treatments. While the antibacterial activity of chitosan is well-known (Sonis et al., 2014; Zivanovic et al., 2007), its specific antibacterial mechanism has not been definitively established. Dina et al. suggested that chitosan's antibacterial ability is regulated by a number of diatheses, which are concentration-dependent and may be related to binding of the tea polyacid and potential extraction of lipids (Xiao et al., 2000; Dina et al., 2008). Other research indicates that the $\mathrm{pH}$, temperature, and the ionic strength in the microenvironment strongly influences chitosan's antibacterial ability. At an acidic $\mathrm{pH}(\mathrm{pH}<6)$ and high temperature $(25-$ $37^{\circ} \mathrm{C}$ ), the increase in ionic strength can significantly improve the sterilizing ability of chitosan, but the metal ions can inhibit the antibacterial effects of chitosan (Guo-Jane and Prot, 1999; Chung et al., 2003). In this study, the antibacterial activity of the CP copolymer system was investigated using E. coli and $S$. aureus (Figure 2). The CP copolymers were transferred to the $E$. coli and $S$. aureus mediums, and the growth status of the colonies near the samples was observed. Bacterial cultures were challenged with three CP copolymer concentration gradients (3.0 to 7.5 $\mathrm{wt} \%)$ to verify the relationship between sample concentration
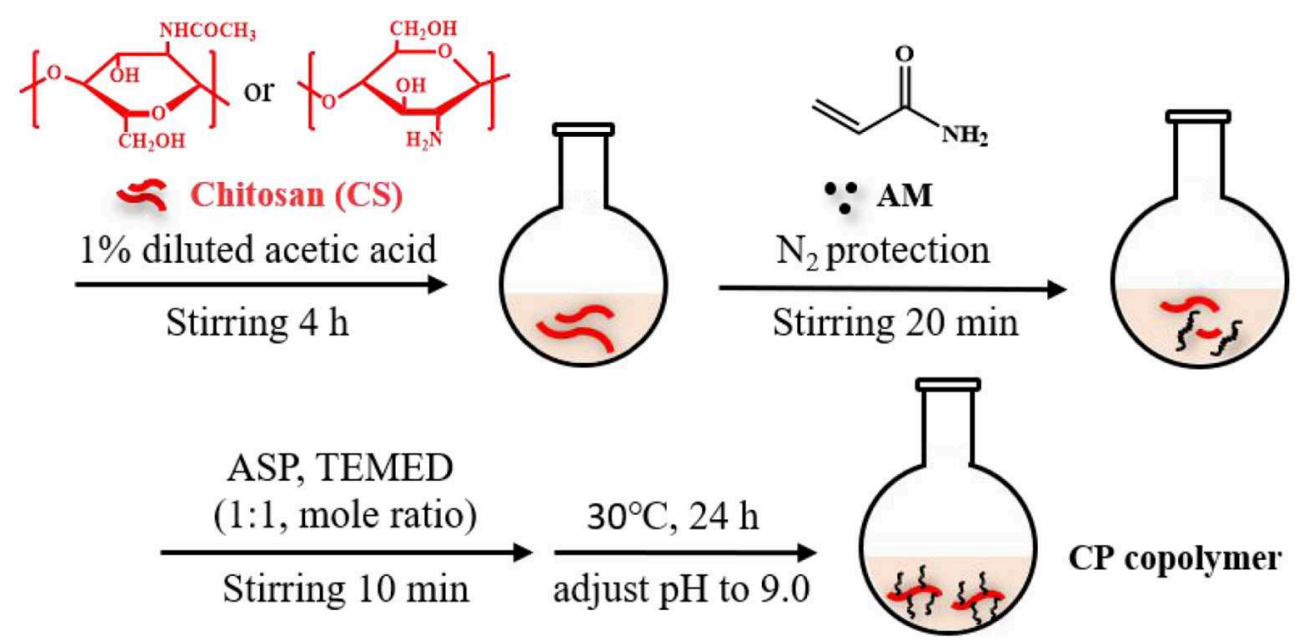

FIGURE 1 | Synthetic scheme of chitosan-based CP copolymer formation by in situ free radical polymerization. 
and antimicrobial activity. It was easy to observe that the $\mathrm{CP}$ copolymer showed antibacterial activity as the antibacterial area $\left(\mathrm{cm}^{2}\right)$ increased with the increasing polymer concentration. The high antibacterial activity of the CP copolymer is likely related to the high specific gravity of chitosan.

\section{CP Copolymer Mediates in vitro Human Gingival Fibroblast Proliferation}

The ability of the CP copolymer to mediate human fibroblast proliferation in the oral cavity was investigated with human gingival fibroblasts (HGF-1), which are often used to study mucosa-related diseases (Häkkinen et al., 2014; Vahabi et al., 2015). The CP copolymer (0 to $1,000 \mu \mathrm{g} / \mathrm{mL}$ ) demonstrated no cytotoxic effects to the HGF-1 cells during $48 \mathrm{~h}$ of culture (Figure 3A). A CCK-8 assay was used to determine the effects of the CP copolymer on HGF-1 cell growth (Figure 3B). Surprisingly, the CP copolymer strongly promoted HGF-1 cell growth. Clearly, the $\mathrm{CP}$ copolymer system significantly promotes cell DNA replication, thereby promoting cell proliferation. The specific gravity of chitosan and PAM caused the increase in HGF-1 cell proliferation in this system.

\section{Hemostatic Effects of the CP Copolymer}

Oral ulcerative lesions are often accompanied by bleeding, which causes patient discomfort and is not conducive to wound healing. Therefore, a hemostatic polymer system is needed to treat oral ulcerative lesion diseases (Lee et al., 2014). Natural, biodegradable chitosan is the only positively charged cationic polysaccharide that can interact with negatively charged red blood cells to form a thrombus (Murugan and Ramakrishna, 2006; Yang et al., 2008). Chitosan can also activate platelets and inhibit the dissolution of fibrin, thereby promoting blood coagulation and hemostasis (Wu et al., 2004; Zigang et al., 2004; Li et al., 2016). To investigate the hemostatic effects of the CP copolymer, blood was collected from Wistar rats (in heparinized EP tubes) by retro-orbital blood sampling. The blood was then mixed with the CP copolymer and incubated in a water bath at $37^{\circ} \mathrm{C}$. As can be seen from (Figure 4A), the $\mathrm{CP}$ copolymer clotted the blood after $30 \mathrm{~s}$. (Figure 4B) shows that the coagulation time of the $\mathrm{CP}$ copolymer was much shorter than that of the control due to the combination of the chitosan skeleton and PAM.

\section{In vivo Therapeutic Effect of the CP Copolymer on Rat Gingival Mucosal Ulcers} As the in vitro experiments showed that the CP copolymer had a promoting effect on the proliferation of human gingival fibroblasts, the in vivo effects of the CP copolymer on oral ulcers were investigated to determine its potential as a therapeutic agent. An oral ulcerative lesion model in Wistar rats was used to investigate the therapeutic effect of the CP copolymer by monitoring the degree of healing every 5 days over a 10-day period (Figure 5A). The swelling area of rats treated with the $\mathrm{CP}$ copolymer after 10 days
A

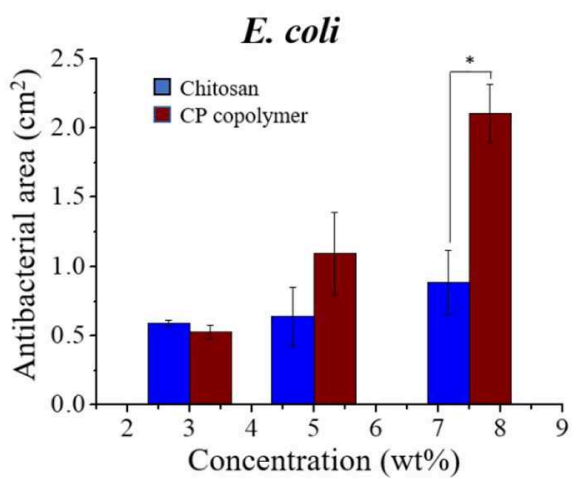

B

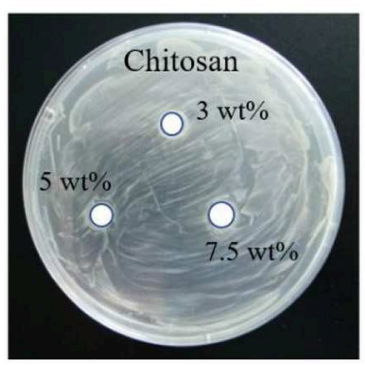

E. coli

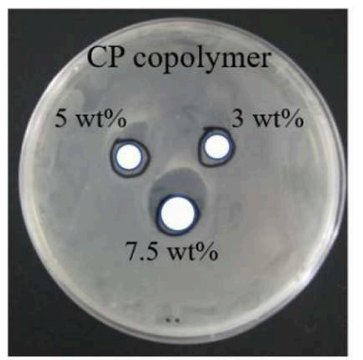

C

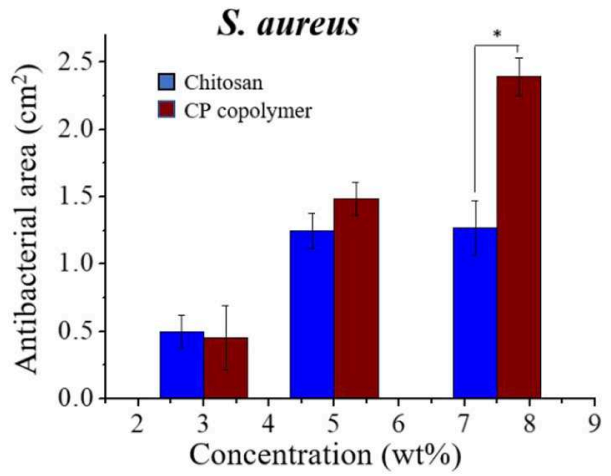

D

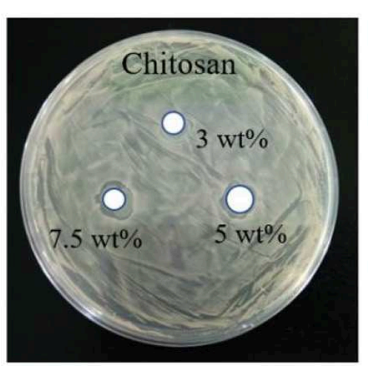

S. aureus

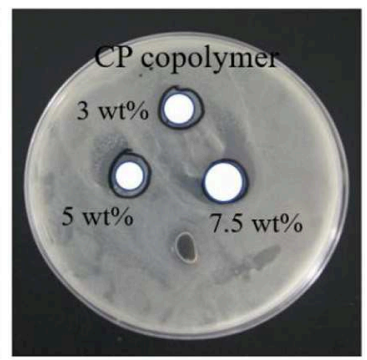

FIGURE 2 | (A) The antibacterial activity of the CP copolymer and chitosan against $E$. coli as determined by the antibacterial area during culture $\left(24 \mathrm{~h}, 37^{\circ} \mathrm{C}\right)$. (B) The antibacterial activity of the CP copolymer and chitosan against $\mathrm{S}$. aureus as determined by the antibacterial area during culture $\left(24 \mathrm{~h}, 37^{\circ} \mathrm{C}\right)$. (C) The quantitative data analysis for (A). (D) The quantitative data analysis for (B). ${ }^{\star} p<0.05$. 
A

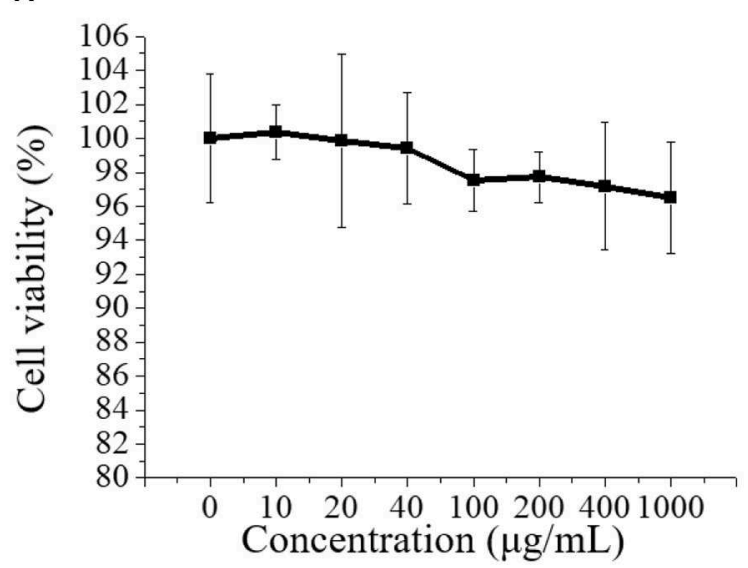

B

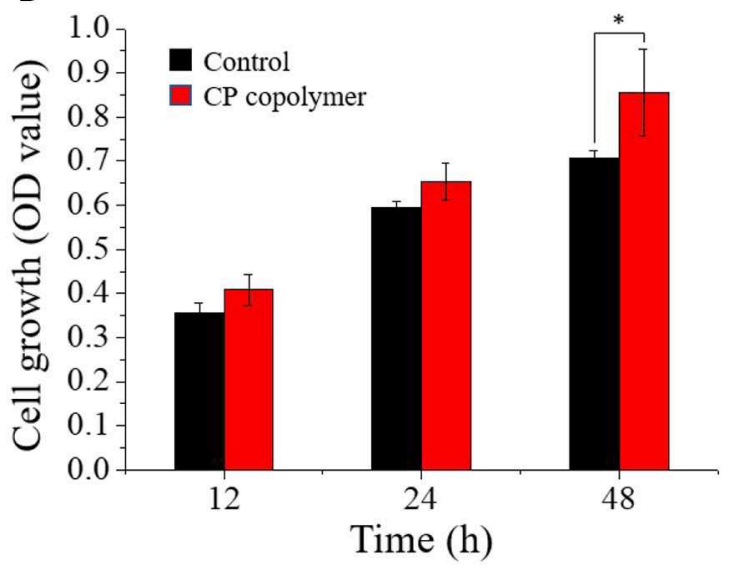

FIGURE 3 | (A) Cell viability of human gingival fibroblast cells treated with the CP copolymer $(0-1,000 \mu \mathrm{g} / \mathrm{mL})$. (B) Cell growth $\left(\mathrm{OD}_{450}\right)$ following the treatment of human gingival fibroblasts with the CP copolymer. ${ }^{*} p<0.05$.
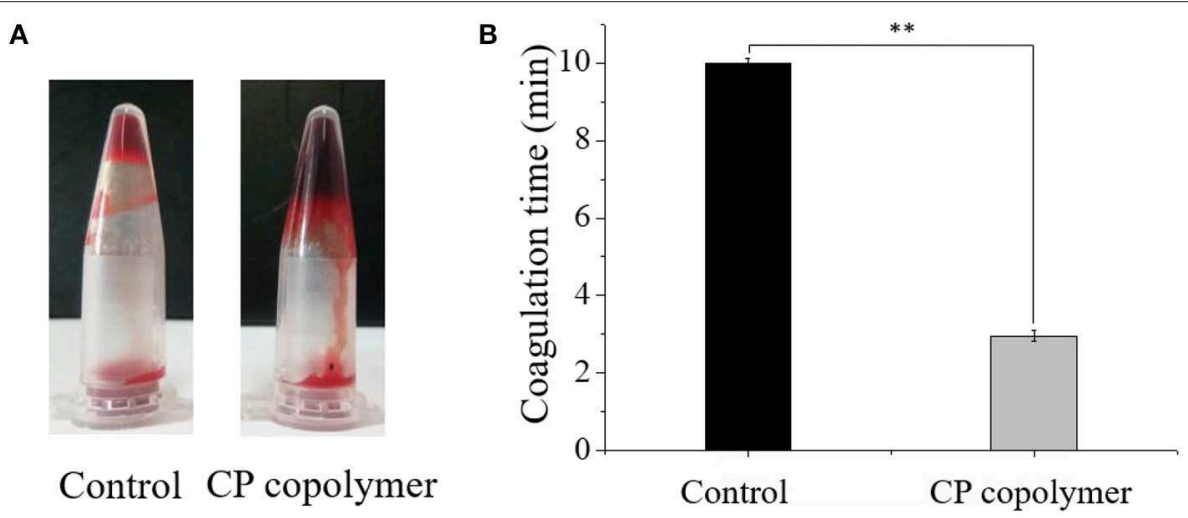

FIGURE 4 | (A) Pictures of blood incubated with the CP copolymer (5 wt\%) in a microcentrifuge tube after $30 \mathrm{~s}$ of treatment in a water bath at $37^{\circ} \mathrm{C}$. (B) Coagulation time was quantified, for which samples from the control group were assigned the maximum clotting time for the assay as they did not clot. ${ }^{\star \star} p<0.01$.
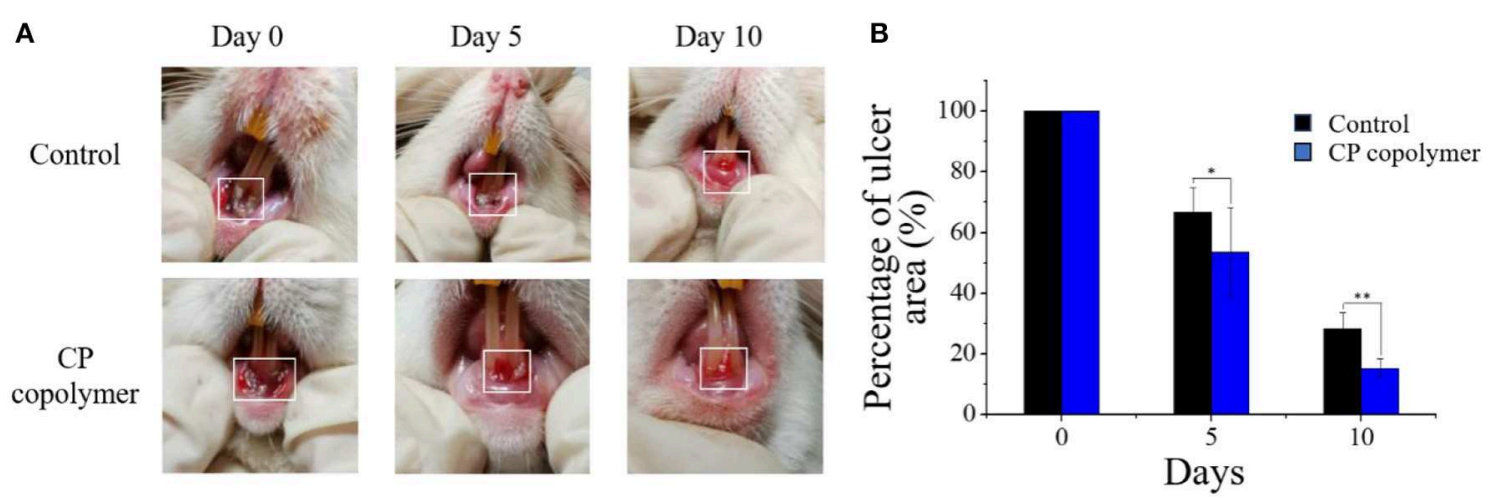

FIGURE 5 | In vivo evaluation of the healing effects of the CP copolymer on oral gingival mucosa ulcers. (A) Photographs of the oral gingival swelling model in Wistar rats treated with the CP copolymer. (B) Gingival swelling areas were measured after dropping normal saline and the CP copolymer onto the ulcerated area. ${ }^{*} p<$ $0.05,{ }^{* *} p<0.01$. 
was much lower than the swelling area of rats in the control group.

(Figure 5B) summarizes the change in the gingival ulcer area over 10 days, which showed that the CP copolymer had a reduction of $46 \%$ in the ulcer area within 5 days, compared to a $33 \%$ reduction in the area in the control group. It is worth mentioning that after 10 days of treatment, the ulcer area in the CP copolymer group decreased by $85 \%$, compared to a $72 \%$ decrease in the control group, indicating that the CP copolymer effectively promoted the healing of oral ulcerative lesions. (Figure 6) shows the change in body weight of the Wistar rats, which was used to further determine the treatment effect on oral ulcerative lesions. It can be seen that the body weight of the five control group rats showed an acute downward trend in the first two days, which may be caused by difficulties in eating due to the induced oral ulcerative lesion. The body weights of rats that recovered after treatment with the CP copolymer were higher than those of rats in the control group, indicating that the $\mathrm{CP}$ copolymer treatment resulted in a gradual reduction of the oral ulceration symptoms and the recovery of feeding. This indicates that

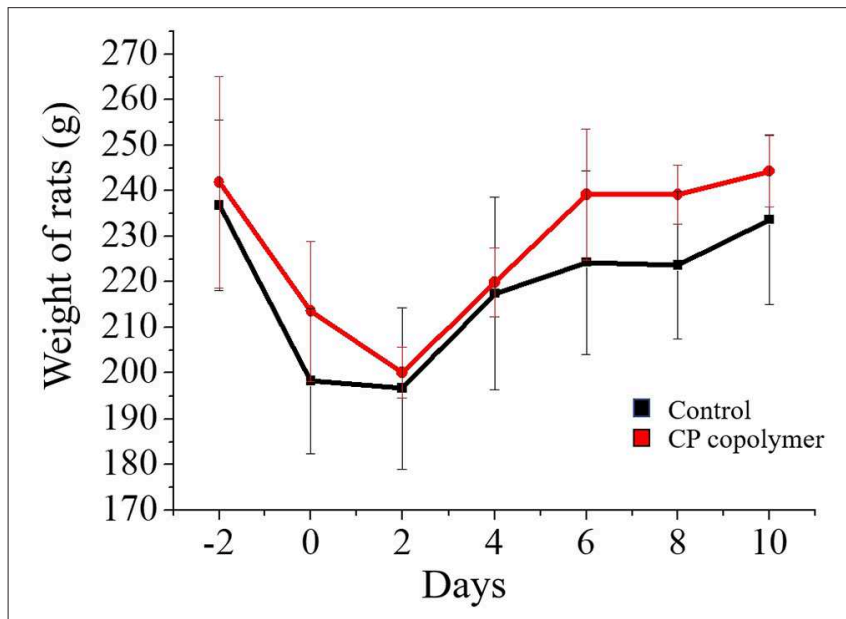

FIGURE 6 | Weight curve of Wistar rats treated with CP copolymer compared to the case for PBS treatment (in the control group). the CP copolymer effectively improved oral festering and promoted wound healing in Wistar rats. The CP copolymer displayed antibacterial effects and simultaneously promoted gingival fibroblast proliferation, making it an effective gingival ulcer treatment.

\section{Histological Sections of Gingival Mucosal Ulcers Treated With the CP Copolymer}

To further investigate the therapeutic effects of the CP copolymer on rat oral gingival mucosa ulcers, histological sections of gingival ulcer sites were analyzed (Figure 7). The gingival ulcer tissue sections were stained with $\mathrm{H} \& \mathrm{E}$ stain, and then observed under a light microscope. Purple-blue staining (hematoxylin) mainly shows chromatin and nucleic acids, and purple-red staining (eosin) shows the extracellular matrix and cytoplasm. In the untreated control group, the cells between the basal layer and the keratinized layer of the epidermis were relatively loose with clear inflammatory cell infiltration. Compared with the control group, the number of newly formed fibroblasts in the CP copolymer group was significantly increased, and the tissue damage was decreased.

\section{CONCLUSIONS}

The biodegradable CP copolymer was successfully synthesized by in situ free-radical polymerization and consisted of a chitosan main chain and PAM polymer side chains. Interestingly, the CP copolymer showed a strong inhibitory effect to Grampositive and Gram-negative bacteria, promoted the proliferation of gingival fibroblasts, displayed significant hemostatic effects, and showed a low cytotoxic effect in vitro. Studies have shown that the inclusion of antibacterial, hemostatic, and proliferative properties are essential in a biomaterial for the treatment of oral ulcerative lesions. More importantly, the CP copolymer showed significant adhesive, anti-inflammatory, and hemostatic effects in the treatment of oral gingival ulcers in rats, indicating that this biodegradable, chitosan-based copolymer is a promising system for development as a therapeutic agent for oral ulcerative lesions.
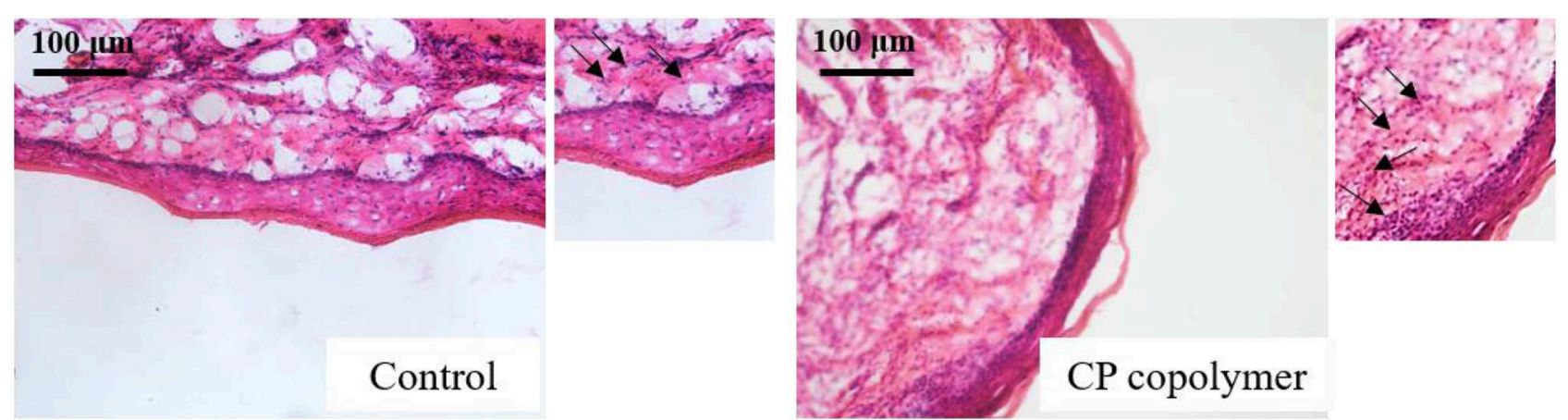

FIGURE 7 | Hematoxylin \& eosin staining of excised oral gingival ulcer tissue sections from Wistar rats after treatment with the control (PBS) or CP copolymer for 10 days. (The black arrows represent fibroblasts). 


\section{DATA AVAILABILITY STATEMENT}

All datasets generated for this study are included in the article/Supplementary Material.

\section{ETHICS STATEMENT}

The animal study was reviewed and approved by the Animal Ethics Committee of Xiamen University.

\section{AUTHOR CONTRIBUTIONS}

YZ, LK, YL, QZ, GD, HW, and XZ conducted the experiments. YZ and LK analyzed the date. YZ, LK, and YL made the draft

\section{REFERENCES}

Berger, J., Reist, M. J., Mayer, J. M. M., Felt, O., and Gurny, R. (2004). Structure and interactions in covalently and ionically cross-linked Chitosan hydrogels for biomedical applications. Eur. J. Pharm. Biopharm. 57, 19-34. doi: 10.1016/S0939-6411(03)00161-9

Bonferoni, M. C., Sandri, G., Rossi, S., Ferrari, F., and Caramella, C. (2009). Chitosan and its salts for mucosal and transmucosal delivery. Expert. Opin. Drug Del. 6, 923-939. doi: 10.1517/17425240903114142

Chung, Y. C., Wang, H. L., and Chen, Y. M. (2003). Effect of abiotic factors on the antibacterial activity of chitosan against waterborne pathogens. Bioresour. Technol. 88, 179-184. doi: 10.1016/S0960-8524(03)00002-6

Dina, R., Kristine, V. B., Albert, H., and Hans-Georg, S. (2008). Insights into the mode of action of chitosan as an antibacterial compound. Appl. Environ. Microbiol. 74, 3764-3773. doi: 10.1128/AEM.00453-08

Echeverria, C., Soares, P., Robalo, A., Pereira, L., Novo, C. M. M., Ferreira, I., et al. (2015). One-pot synthesis of dual-stimuli responsive hybrid PNIPAAm-chitosan microgels. Mater. Design. 86, 745-751. doi: 10.1016/j.matdes.2015.07.170

Elsabee, M. Z., Morsi, R. E., and Al-Sabagh, A. M. (2009). Surface active properties of chitosan and its derivatives. Colloids Surf. B Biointerfaces. 74, 1-16. doi: 10.1016/j.colsurfb.2009.06.021

Feller, L., Essop, R., Wood, N. H., Khammissa, R. A. G., and Lemmer, J. (2010). Chemotherapy- and radiotherapy-induced oral mucositis: pathobiology, epidemiology and management. SADJ 65, 372-374.

Guo-Jane, T., and Prot, S. W. H. (1999). Antibacterial activity of shrimp chitosan against Escherichia coli. J. Food. Protect. 62, 239-243. doi: 10.4315/0362-028X-62.3.239

Häkkinen, L., Larjava, H., and Fournier, B. (2014). Distinct phenotype and therapeutic potential of gingival fibroblasts. Cytotherapy. 16, 1171-1186. doi: 10.1016/j.jcyt.2014.04.004

Huang, C. H., Wang, C. F., and Don, T. M. (2013). Preparation of pH- and thermosensitive chitosan-PNIPAAm core-shell nanoparticles and evaluation as drug carriers. Cellulose. 20, 1791-1805. doi: 10.1007/s10570-013-9951-1

Kalambettu, A. B., and Rajangam, P. (2012). The effect of chlorotrimethylsilane on bonding of nano hydroxyapatite with a chitosan-polyacrylamide matrix. Carbohyd. Res. 352, 143-150. doi: 10.1016/j.carres.2011. 12.027

Kandow, C. E., Georges, P. C., Janmey, P. A, and Beningo, K. A. (2007). Polyacrylamide hydrogels for cell mechanics: steps toward optimization and alternative uses. Methods Cell Biol. 83, 29-46. doi: 10.1016/S0091-679X(07)83002-0

Karim, A. A., Dou, Q., and Li, Z. (2016). Emerging supramolecular therapeutic carriers based on host-guest interactions. Chem. Asian J. 11, 1300-1321. doi: 10.1002/asia.201501434

Lee, D. Y., Kim, H. B., Shim, I. K., Kanai, N., and Okano, T. (2016). Treatment of chemically induced oral ulcer using adipose-derived mesenchymal stem cell sheet. J. Oral. Pathol. Med. 46, 520-527. doi: 10.1111/jop.12517 and finalized this manuscript. YZ and LK contributed equally to this work.

\section{FUNDING}

Funding was provided by the Xiamen Science and Technology Program Grant (Grant No. 3502Z20174025) and the Xiamen Medical College Science Foundation (Grant No. K2017-03).

\section{SUPPLEMENTARY MATERIAL}

The Supplementary Material for this article can be found online at: https://www.frontiersin.org/articles/10.3389/fchem. 2020.00273/full\#supplementary-material

Lee, S., Jung, I., Yu, S., and Hong, J. (2014). Effect of recombinant human epidermal growth factor impregnated chitosan film on hemostasis and healing of blood vessels. Arch. Plast. Surg. 41, 466-471. doi: 10.5999/aps.2014.41.5.466

Lewandowska, K. (2007). Comparative studies of rheological properties of polyacrylamide and partially hydrolyzed polyacrylamide solutions. J. Appl. Polym. Sci. 103, 2235-2241. doi: 10.1002/app.25247

Lewandowska, K. (2015). Liquids KJJoM. Miscibility and physical properties of chitosan and polyacrylamide blends. J. Mol. Liq. 209, 301-305. doi: 10.1016/j.molliq.2015.05.049

Li, J., Wei, C. P., Wang, F. M., Dong, L. D., Sun, S., and Liu, Q. Y. (2016). Preparation and performance research of hemostatic powder of chitosan mixed with scutellaria baicalensis. Mater. Sci. Forum. 852, 1277-1281. doi: 10.4028/www.scientific.net/MSF.852.1277

Lu, Z.,Gao, J., He, Q., Wu, J., Liang, D., Yang, H., et al. (2017). Enhanced antibacterial and wound healing activities of microporous chitosan-Ag/ZnO composite dressing. Carbohydr. Polym. 156, 460-469. doi: 10.1016/j.carbpol.2016.09.051

Mahattanadul, S., Mustafa, M., Kuadkaew, S., Pattharachayakul, S., Ungphaiboon, S., and Sawanyawisuth, K. (2018). Oral ulcer healing and anti-candida efficacy of an alcohol-free chitosan-curcumin mouthwash. Eur. Rev. Med. Pharmacol. Sci. 22, 7020-7023. doi: 10.26355/eurrev_201810_16173

Martín, M. J., Calpena, A. C., Fernández, F., Mallandrich, M., and Gálvez, P. (2015). Development of alginate microspheres as nystatin carriers for oral mucosa drug delivery. Carbohyd. Polym. 117, 140-149. doi: 10.1016/j.carbpol.2014.09.032

McGuire, D. B., Fulton, J. S., Park, J., Brown, C. G., Correa, M. E. P., Eilers, J., et al. (2013). Systematic review of basic oral care for the management of oral mucositis in cancer patients. Support Care Cancer. 21, 3165-3177. doi: 10.1007/s00520-013-1942-0

Murugan, R., and Ramakrishna, S. (2006). Production of ultra-fine bioresorbable carbonated hydroxyapatite. Acta. Biomater. 2, 201-206. doi: 10.1016/j.actbio.2005.09.005

Nettles, D. L., and Elder, S. H. (2002). Potential use of chitosan as a cell scaffold material for cartilage tissue engineering. Tissue Eng. 8, 1009-1016. doi: 10.1089/107632702320934100

Raber-Durlacher, J. E., Logan, R. M., Bowen, J., Al-Azri, A. R., Everaus, H., Gerber, E., et al. (2013). Systematic review of cytokines and growth factors for the management of oral mucositis in cancer patients. Supp. Care Cancer. 21, 343-355. doi: 10.1007/s00520-012-1594-5

Risbud, M. V., and Bhonde, R. (2000). Polyacrylamide-chitosan hydrogels: in vitro biocompatibility and sustained antibiotic release studies. Drug. Deliv. 7, 69-75. doi: $10.1080 / 107175400266623$

Shao, Y., and Zhou, H. (2019). Clinical evaluation of an oral mucoadhesive film containing chitosan for the treatment of recurrent aphthous stomatitis: a randomized, double-blind study. J. Dermatol. Treat. 1-5. doi: 10.1080/09546634.2019.1610548

Sheng, Q., Tian, W., Lapierre, F., Gao, S., Mulder, R. J., and Zhu, Y., et al. (2014). Arrays of polyacrylamide hydrogels using a carbodiimide-mediated crosslinking reaction. J. Appl. Polymer Sci. 131, 1-11. doi: 10.1002/app.40416 
Sonis, S. T., Elting, L. S., Keefe, D., Peterson, D. E., Schubert, M., Hauer-Jensen, M., et al. (2014). Perspectives on cancer therapy-induced mucosal injury: pathogenesis, measurement, epidemiology, and consequences for patients. Cancer. 100, 1995-2025. doi: 10.1002/cncr.20162

Sumita, Y., Minamizato, T., Umebayashi, M., Liu, Y., Tran, S. D., and Asahina, I. (2014). Bone marrow-derived cell therapy for oral mucosal repair after irradiation. J. Dental Res. 93, 813-820. doi: 10.1177/0022034514 541124

Trautwein-Weidner, K., Gladiator, A., Nur, S., Diethelm, P., and LeibundGutLandmann, S. (2015). IL-17-mediated antifungal defense in the oral mucosa is independent of neutrophils. Mucosal Immunol. 8, 221-231. doi: $10.1038 / \mathrm{mi} .2014 .57$

Vahabi, S., Vaziri, S., Torshabi, M., and Esfahrood, Z. (2015). Effects of plasma rich in growth factors and platelet-rich fibrin on proliferation and viability of human gingival fibroblasts. J. Dent. 12, 504.

Vieira, E. L., Leonel, A. J., Sad, A. P., Beltrão, N. R., Costa, T. F., and Ferreira, T. M. (2012). Oral administration of sodium butyrate attenuates inflammation and mucosal lesion in experimental acute ulcerative colitis. J. Nutr. Biochem. 23, 430-436. doi: 10.1016/j.jnutbio.2011.01.007

Wang, J., and Wang, H. (2011). Preparation of soluble p-aminobenzoyl chitosan ester by Schiff's base and antibacterial activity of the derivatives. Int. J. Biol. Macromol. 48, 523-529. doi: 10.1016/j.ijbiomac.2011.01.016

Wang, W., and Yu, W. (2015). Preparation and characterization of CS-gPNIPAAm microgels and application in a water vapour-permeable fabric. Carbohyd. Polym. 127, 11-18. doi: 10.1016/j.carbpol.2015.03.040

Warinner, C., Rodrigues, J. F. M., Vyas, R., Trachsel, C., Shved, N., and Grossmann, J. (2014). Pathogens and host immunity in the ancient human oral cavity. Nat. Genet. 46, 336-344. doi: 10.1038/ng.2906

Wilkinson, L. J., White, R. J., and Chipman, J. K. (2011). Silver and nanoparticles of silver in wound dressings: a review of efficacy and safety. J. Wound Care. 20, 543-549. doi: 10.12968/jowc.2011.20.11.543

Wu, T. J., Huang, H. H., Lan, C. W., Lin, C. H., and Hsu, F. Y. (2004). Studies on the microspheres comprised of reconstituted collagen and hydroxyapatite. Biomaterials. 25, 651-658. doi: 10.1016/S0142-9612(03)00576-3

Xiao, F. L., Yun, L. G., Dong, Z. Y., Zhi, L., and Kang, D. (2000). Antibacterial action ofchitosan and carboxymethylated chitosan. J. Appl. Polym. Sci.
79:1324-1335. doi: 10.1002/1097-4628(20010214)79:7>1324::AID-APP210< 3.0.CO;2-L

Yang, J., Tian, F., Wang, Z., Wang, Q., Zeng, Y. J., and Chen, S. Q. (2008). Effect of chitosan molecular weight and deacetylation degree on hemostasis. J. Biomed. Mater. Res. B Appl. Biomater. 84, 131-137. doi: 10.1002/jbm.b.30853

Zhang, C., Hou, H., and Li, J. (2019). Chitosan/matrine membrane preparations promote wound recovery in an in vivo animal model of ulcer trauma. Int. J. Clin. Exp. Med. 12, 504-512.

Zhang, Y., Wang, S., Eghtedari, M., and Motamedi, M. (2005). Inverted-colloidalcrystal hydrogel matrices as three-dimensional cell scaffolds. Adv. Funct. Mater. 15, 725-731. doi: 10.1002/adfm.200400325

Zhang, Z. X., Liow, S. S., Xue, K., Zhang, X., Li, Z., and Loh, X. J. (2019). Autonomous chitosan-based self-healing hydrogel formed through noncovalent interactions. ACS Appl. Polym. Mater. 1, 1769-1777. doi: 10.1021/acsapm.9b00317

Zigang, G., Sophie, B., Lee, Y. L., Aileen, W., and Eugene, K. (2004). Hydroxyapatite-chitin materials as potential tissue engineered bone substitutes. Biomaterials. 25, 1049-1058. doi: 10.1016/S0142-9612(03)00612-4

Zivanovic, S., Li, J., Davidson, P. M., and Kit, K. J. B. (2007). Physical, mechanical, and antibacterial properties of chitosan/PEO blend films. Biomacromolecules. 8, 1505-1510. doi: 10.1021/bm061140p

Conflict of Interest: The authors declare that the research was conducted in the absence of any commercial or financial relationships that could be construed as a potential conflict of interest.

The reviewer YW declared a shared affiliation, though no other collaboration, with one of the authors LK to the handling editor.

Copyright (c) 2020 Zheng, Ke, Lu, Zuo, Deng, Wang and Zeng. This is an open-access article distributed under the terms of the Creative Commons Attribution License (CC BY). The use, distribution or reproduction in other forums is permitted, provided the original author(s) and the copyright owner(s) are credited and that the original publication in this journal is cited, in accordance with accepted academic practice. No use, distribution or reproduction is permitted which does not comply with these terms. 